\title{
Vav3 oncogene activates estrogen receptor and its overexpression may be involved in human breast cancer Kiwon Lee ${ }^{1}$, Yin Liu ${ }^{1}$, Jun Qin Mo ${ }^{1}$, Jinsong Zhang ${ }^{2}$, Zhongyun Dong ${ }^{3}$ and Shan $\mathrm{Lu}^{* 1}$
}

Address: ${ }^{1}$ Department of Pathology, University of Cincinnati College of Medicine, 2120 E. Galbraith Road, Cincinnati, OH 45237, USA, 2Department of Cancer and Cell Biology, University of Cincinnati College of Medicine, 231 Albert Sabin Way, Cincinnati, OH 45267, USA and ${ }^{3}$ Department of Medicine, University of Cincinnati College of Medicine, 231 Albert Sabin Way, Cincinnati, OH 45267, USA

Email: Kiwon Lee - leeko@UCMAIL.UC.EDU; Yin Liu - yin.liu@uc.edu; Jun Qin Mo - Jun.Mo@cchmc.org; Jinsong Zhang - zhangj5@ucmail.uc.edu; Zhongyun Dong - dongzu@ucmail.uc.edu; Shan Lu* - shan.lu@uc.edu

* Corresponding author

Published: 2 June 2008

BMC Cancer 2008, 8:158 doi:10.1/86/147|-2407-8-158
Received: 18 January 2008

Accepted: 2 June 2008

This article is available from: http://www.biomedcentral.com/I47I-2407/8/I58

(C) 2008 Lee et al; licensee BioMed Central Ltd.

This is an Open Access article distributed under the terms of the Creative Commons Attribution License (http://creativecommons.org/licenses/by/2.0), which permits unrestricted use, distribution, and reproduction in any medium, provided the original work is properly cited.

\begin{abstract}
Background: Our previous study revealed that Vav3 oncogene is overexpressed in human prostate cancer, activates androgen receptor, and stimulates growth in prostate cancer cells. The current study is to determine a potential role of Vav3 oncogene in human breast cancer and impact on estrogen receptor a $(E R \alpha)$-mediated signaling axis.
\end{abstract}

Methods: Immunohistochemistry analysis was performed in 43 breast cancer specimens and western blot analysis was used for human breast cancer cell lines to determine the expression level of Vav3 protein. The impact of Vav3 on breast cancer cell growth was determined by siRNA knockdown of Vav3 expression. The role of Vav3 in ER $\alpha$ activation was examined in luciferase reporter assays. Deletion mutation analysis of Vav3 protein was performed to localize the functional domain involved in ER $\alpha$ activation. Finally, the interaction of $\operatorname{Vav} 3$ and ER $\alpha$ was assessed by GST pull-down analysis.

Results: We found that Vav3 was overexpressed in $81 \%$ of human breast cancer specimens, particularly in poorly differentiated lesions. Vav3 activated ER $\alpha$ partially via PI3K-Akt signaling and stimulated growth of breast cancer cells. Vav3 also potentiated EGF activity for cell growth and ER $\alpha$ activation in breast cancer cells. More interestingly, we found that Vav3 complexed with ER $\alpha$. Consistent with its function for AR, the DH domain of $\operatorname{Vav} 3$ was essential for ER $\alpha$ activation.

Conclusion: Vav3 oncogene is overexpressed in human breast cancer. Vav3 complexes with ER $\alpha$ and enhances ER $\alpha$ activity. These findings suggest that Vav3 overexpression may aberrantly enhance ER $\alpha$-mediated signaling axis and play a role in breast cancer development and/or progression.

\section{Background}

Vav3 oncogene, a quanine nucleotide exchange factor (GEF) for Rho family GTPases, belongs to Vav family pro- teins. The three mammalian Vav proteins (Vav1, Vav2, and Vav3) differ in their tissue distribution. Vav1 is primarily expressed in hematopoietic cells, while Vav2 and Vav3 
are more ubiquitously expressed [1,2]. Vav proteins contain multiple function motifs and are involved in various cellular signaling processes, including cytoskeleton organization, calcium influx, phagocytosis, and cell transformation [3]. Vav proteins share a common structure, including a N-terminal calponin homology $(\mathrm{CH})$ domain involved in $\mathrm{Ca}^{+2}$ mobilization and transforming activity, an acidic domain (AD) containing three regulatory tyrosines, a Dbl homology (DH) domain with a conserved region that promotes the exchange of GDP for GTP on Rac/Rho GTPases, a pleckstrin homology (PH) domain binding to $\mathrm{PIP}_{3}$ that enables its movement to the inner face of the plasma membrane, two Src-homology 3 (SH3) domains interacting with proteins containing proline-rich sequences, and a Src-homology 2 (SH2) domain interacting with proteins containing phosphorylated tyrosines $[4,5]$. Tyrosine phosphorylation by receptor protein tyrosine kinase or cytoplasmic protein tyrosine kinase is required for Vav protein activation. In the non-phosphorylation state, Vav is folded, which is achieved by binding of the tyrosines in the $\mathrm{AD}$ domain to the $\mathrm{DH}$ domain and binding of the $\mathrm{CH}$ domain to the $\mathrm{C} 1$ region. Upon phosphorylation of the tyrosines in the AD domain, the folding is opened and the DH domain is exposed. Thus, Vav protein is activated and interacts with substrate proteins, and the PH domain is exposed for PIP3 binding [6].

Breast cancer is the most common malignant disease worldwide and the number one cause of cancer-related death among non-smoking women in the US. The major problem in breast cancer therapy is development of estrogen-insensitive growth after hormonal therapy. Aberrant $\mathrm{ER} \alpha$ activation by various mechanisms contributes to breast cancer development and estrogen-resistant diseases [7-9]. This ER $\alpha$ hypersensitivity can be achieved by estrogen-independent mechanisms, such as ER $\alpha$ phosphorylation by crosstalking with signal transduction pathways and overexpression of nuclear receptor coactivator SRC3 $[10,11]$. Numerous studies have shown that EGFR/HER2elicited signaling is involved in human breast cancer [9]. In addition, elevated PI3K-Akt signaling, mediated by PTEN deletion and/or mutation and PI3K subunit p110a (PI3KCA) mutation, upregulates ER $\alpha$ activity and is correlated with the breast cancer development and anti-estrogen resistance [12-15]. Activation of PI3K has been implicated in part because the downstream PI3K target, Akt, phosphorylates and promotes ligand-independent ER $\alpha$ activation $[16,17]$. Transgenic breast cancer mouse models have confirmed that elevated signaling in the EGFR/HER2-PI3K-Akt pathway either by targeted Akt overexpression or HER2 overexpression in breast epithelial cells induces breast cancer development [18-20]. These signaling pathways have been the targets for breast cancer therapy [7-9].
The classical ER $\alpha$ is a ligand-dependent transcription factor that activates transcription of its target genes in nucleus, which is known as genomic ER $\alpha$ activity. Recent findings revealed that the classical steroid hormone receptors also associate with cell membrane and mediate cell signaling through kinase cascade, defined as nongenomic activity $[21,22]$. Nongenomic ER $\alpha$ resides in multiprotein complexes with molecules, such as MNAR/PELP1 and src, in the cytoplasm and signals through the PI3K-Akt and MAPK pathways in breast cancer cells [23,24]. Nongenomic ER $\alpha$ signaling has been shown to contribute to estrogen-independent growth in breast cancer.

Recently, we and others found that Vav3 oncogene is overexpressed in androgen-independent prostate cancer cells, enhances androgen receptor (AR) activity, and stimulates androgen-independent growth in prostate cancer cells $[25,26]$. We further showed that Vav3, as a signal transducer, upregulates AR activity partially via PI3K-Akt signaling [25]. The DH domain of Vav3 is responsible for AR activation. Vav3 also potentiates EGF activity for cell growth and AR activation in prostate cancer cells. More importantly, Vav3 is overexpressed in $32 \%$ of human prostate cancer. These findings suggest that Vav3 overexpression may be involved in prostate cancer.

The purpose of this study is to determine the role of Vav3 in breast cancer. We found that Vav3 is overexpressed in human breast cancer specimens and cell lines. Vav3 stimulates growth of breast cancer cells and activates ER $\alpha$ partially via PI3K-Akt signaling. Vav3 potentiates EGF activity for cell growth and ER $\alpha$ activation in breast cancer cells. These data suggest that Vav3 impacts on ER $\alpha$ signaling axis and its overexpression may be involved in breast cancer.

\section{Methods \\ Reagents}

RPMI 1640 medium was purchased from Invitrogen (Gaithersburg, MD). Fetal bovine serum (FBS) and charcoal/dextran-treated FBS were purchased from HyClone Laboratories (Logan, UT). Human Vav3-specific Stealth ${ }^{\mathrm{TM}}$ Select RNAi (siVav3-247: 5'-CCCAGTTTCTCTGTTTGAAGAACAT-3') and its control (control-247: 5'-CCCTTCTCTGTTTGTAAAGAGACAT-3') were designed by a software in Invitrogen website and we purchased both oligos from Invitrogen as described before [25]. The transfection reagent Lipofectamine ${ }^{\mathrm{TM}} 2000$ was from Invitrogen. Anti-Vav3 and anti-ER $\alpha$ antibodies were obtained from Upstate Biotechnology (Charlottesville, VA).

\section{Cell culture}

The human breast cancer cell lines MCF7 and T47D, and cervical carcinoma cell line Hela were obtained from ATCC (Rockville, MD) and maintained in RPMI-1640 
medium supplemented with 10\% FBS (complete medium) at $37^{\circ} \mathrm{C}$ in 5\% CO2. Nontumoral breast epithelial MCF-10A cells were obtained from ATCC. Transient transfection experiments were performed in RPMI-1640 medium supplemented with $10 \%$ charcoal/dextrantreated FBS (stripped medium).

\section{Plasmids}

Plasmids pBEF-Vav3, pHEF-Vav3*, pHEF-Vav3*- $\triangle \mathrm{DH}$, pHEF-Vav3 $*-\Delta S H$, and control vector $\mathrm{pHEF}$ were detailed in our previous studies [25]. pS2-Luc is a gift from Dr. Sohaib Khan, Department of Cell Biology, University of Cincinnati College of Medicine. ER $\alpha$ and ERE-Luc are gifts from Dr. Zafar Nawaz, Braman Breast Cancer Institute, University of Miami Miller School of Medicine.

For generation of GST-Vav3-DH+PH construct, we designed upper primer 5' CGAGAATTCAAGGCAGAGGAAGCACATCAG containing Eco RI site and lower primer 5' TCTGCGGCCGCTGTTTAGGAGTTCTTCGCAG containing Not I site flanking both the $\mathrm{DH}$ and $\mathrm{PH}$ domains of Vav3 gene. The PCR product by amplification of the DH and $\mathrm{PH}$ domains using this pair of primers was subcloned into pGEX-4T-1 vector (GE Healthcare Bio-Sciences Corp. Piscataway, NJ) in frame by Eco RI and Not I sites.

\section{Cell growth assay}

Tumor cell growth was estimated by MTT assay as previously described [27]. Briefly, breast cancer cells were seeded into 96-well cell culture plates at a density of $2.5 \times$ $10^{3}$ cells/well in stripped medium. After incubation in 5\% $\mathrm{CO} 2$ at $37^{\circ} \mathrm{C}$ overnight, the cells were transfected with Vav3 siRNA and control siRNA using Lipofectamine 2000 and then cultured in stripped medium without or with E2 $\left(10^{-9} \mathrm{M}\right)$ for 5 days. At the end of incubation, $20 \mathrm{ul}$ of MTT $(2.5 \mathrm{mg} / \mathrm{ml}$ in PBS) was added to each well, and the cells were further incubated for one hour at $37^{\circ} \mathrm{C}$ to allow complete reaction between the dye and the enzyme mitochondrial dehydrogenase in the viable cells. After removal of the residual dye and medium, $100 \mathrm{ul}$ of dimethylsulfoxide was added to each well, and the absorbance at 570 nm was measured using BMG microplate Reader (BMG Labtech, Inc., Durham, NC).

\section{Western blot analysis}

Western blot analysis was performed as previously described [27]. Briefly, aliquots of samples with the same amount of protein, determined using the Bradford assay (BioRad, Hercules, CA), were mixed with loading buffer (final concentrations of $62.5 \mathrm{mM}$ Tris- $\mathrm{HCl}, \mathrm{pH} 6.8,2.3 \%$ SDS, $100 \mathrm{mM}$ dithiothreitol, and $0.005 \%$ bromophenol blue), boiled, fractionated in a SDS-PAGE, and transferred onto a 0.45 -um nitrocellulose membrane (BioRad). The filters were blocked with $2 \%$ fat-free milk in PBS, and probed with first antibody in PBS containing $0.1 \%$ Tween 20 (PBST) and 1\% fat-free milk. The membranes were then washed four times in PBST and incubated with horseradish peroxidase-conjugated secondary antibody (BioRad) in PBST containing 1\% fat-free milk. After washing four times in PBST, the membranes were visualized using the ECL Western blotting detection system (Amersham Co., Arlington Height, IL). For western blot analysis of Vav3 expression, the first antibody was incubated overnight at $4^{\circ} \mathrm{C}$.

\section{Reporter assay}

Cells $\left(10^{5} /\right.$ well) were seeded in 12 well tissue culture plates. Next day, Optifect-mediated transfection was used for the transient transfection assay according to the protocol provided by Invitrogen/Life Technologies, Inc. The cells were then treated with hormone or drugs in stripped medium for 24 hours. Subsequently, the cell extracts were prepared and luciferase activity was assessed in a Berthold Detection System (Pforzheim, Germany) using a kit (Promega, Madison, WI) following the manufacture's instruction. For each assay, cell extract (20 ul) was used and the reaction was started by injection of $50 \mathrm{ul}$ of luciferase substrate. Each reaction was measured for $10 \mathrm{sec}-$ onds in the Luminometer. Luciferase activity was defined as light units/mg protein.

\section{GST pull down}

GST-Vav3-DH+PH and control GST vectors were transformed into BL21 bacteria, respectively (Protein Express, Inc. Cincinnati, $\mathrm{OH}$ ). The transformed bacteria were cultured in L-Broth with addition of $100 \mathrm{uM}$ of IPTG to induce GST-fusion protein expression. Then, the bacteria were harvested and subjected to GST fusion protein purification by Sonication and using Glutathione Sepharose 4B (Amersham Bioscience).

For pull down reaction, 5 10 ug of GST or GST-Vav3$\mathrm{DH}+\mathrm{PH}$ was incubated with $1 \mathrm{mg}$ of cell extracts from MCF7 cells in a binding buffer [20 mM of Tris.CL, PH. 7.9; $300 \mathrm{mM}$ of KCL; $0.05 \%$ of NP-40; 0.2 mM of EDTA; $20 \%$ of Glycerol; $1 \mathrm{mM}$ of Dithiothritol; $1 \mathrm{mM}$ of phenylmethylsulfonyl fluoride (PMSF), $1 \times$ of protease inhibitor cocktail (Roche Diagnostics)] for overnight $[28,29]$. Then, the beads were washed for five times in a washing buffer (20 mM of Tris.CL, PH. 7.9; $300 \mathrm{mM}$ of NaCL; $0.01 \%$ of NP$40 ; 0.2 \mathrm{mM}$ of EDTA; $20 \%$ of Glycerol; $0.5 \mathrm{mM}$ of Dithiothritol) and boiled in $1 \times$ SDS loading buffer. The proteins in the supernatant were subjected for SDS-PAGE, which was visualized by Coomassie Blue staining. The samples were also subjected to western blot analysis for $\mathrm{ER} \alpha$. 


\section{Immunohistochemistry (IHC) staining}

IHC staining was performed as detailed in our previous studies [25]. Briefly, paraffin-embedded section of breast cancer tissue array (US Biomax, Rockville, MD) was deparaffinized in xylene, rehydrated in graded alcohol, and transferred to PBS. The slides were treated with a citric acid-based antigen-retrieval buffer (DAKO Co., Carpinteria, CA), followed by $3 \% \mathrm{H}_{2} \mathrm{O}_{2}$ in methanol, incubated in blocking buffer (5\% BSA and 5\% horse serum in PBS) and then in the blocking buffer containing antibodies against human Vav3 (Upstate Biotechnology Inc.). After washing, the slide was incubated with a biotinylated secondary antibody (BioGenex Laboratories, San Ramon, CA), followed by washing and incubation with the streptavidinconjugated peroxidase (BioGenex). A positive reaction was visualized by incubating the slides with stable diaminobenzidine and counterstaining with Gill's hematoxylin (BioGenex) and mounted with Universal Mount mounting medium (Fisher Scientific, Pittsburgh, PA). The intensity and extent of cytoplasm-positive labeling for Vav3 in tissue arrays were assessed semiquantitatively and scored as 0 (no staining), $1+$ (weak and focal staining in $<25 \%$ of tissue), $2+$ (moderate intensity in $25-50 \%$ of tissue), and $3+$ (moderate intensity in $>50 \%$ of tissue), and $4+$ (strong and diffused staining in $>50 \%$ of tissue).

\section{Results \\ Expression analysis of Vav3 in human breast cancer specimens}

To determine a potential role of Vav3 in breast cancer, we evaluated Vav3 expression in surgical specimens of human breast cancer by IHC analysis using anti-Vav3 antibody. No significant Vav3 immunoreactivity was detected in all normal breast tissue sections $(0 / 8)$ (Table 1 and Figure 1). In contrast, Vav3 staining, detected in both cytoplasm and nucleus of the epithelial cells but not in stroma of breast tissues, was found in 35 out of 43 tumor tissue sections $(35 / 43,81 \%, p<0.0001)$. Among the tumor sections, Vav3 staining was found in approximately $67 \%$ $(27 / 35)$ of the specimens with well to moderately differentiated tumors and $100 \%(8 / 8)$ of those with poorly dif-

Table I: IHC analysis of Vav3 expression in breast cancer specimens.

\begin{tabular}{lcccc}
\hline Differentiation & \multicolumn{3}{c}{ Staining intensity } & Intensity $(>1)$ \\
\hline & Negative & $1-2$ & $3-4$ & \\
& 8 & 22 & 5 & $27 / 35$ \\
Well to moderate & 0 & 2 & 6 & $\begin{array}{c}8 / 8 \\
0 / 8\end{array}$ \\
Poor & 8 & 0 & 0 & $\mathrm{P}<0.000 \mathrm{I}$ \\
Normal breast tissue & & & & $\mathrm{P}>0.05$ \\
Normal vs. tumor & & &
\end{tabular}

Differences in the IHC staining of surgical specimens of human breast cancer were analyzed with the chi-square test. ferentiated tumors, respectively. The statistical analysis of association of Vav3 overexpression with poorly differentiated breast tumors failed ( $p>0.05)$, possibly due to the small number of poorly differentiated tumor specimens. The proportion of sections with higher intensity of Vav3 staining, however, was significantly elevated in the specimens with poorly differentiated tumors $(p<0.01)$. As shown in Figure 1, for moderately and poorly differentiation breast cancer cells, the nuclei are significantly enlarged, hyperchromatic with coarse clumping of chromatin, prominent nucleoli, and irregular nuclear membrane.

Immunoblotting analysis also revealed that Vav3 expression was elevated in MCF-7 and T47D breast cancer cells in comparison with that in nontumoral breast epithelial MCF-10A cells (Figure 2A). ER $\alpha$ was detected in the two breast cancer cell lines, but not at the very low level in nontumoral MCF-10A cells.

\section{Vav3 is involved in growth of breast cancer cells}

To determine a potential role of Vav3 in growth of breast cancer cells, we determined the role of Vav3 on estrogenindependent growth in breast cancer cells. Control and Vav3-transfected MCF7 cells were incubated in stripped medium in the presence of increasing concentrations of EGF. We found that Vav3 overexpression stimulated MCF7 cell growth in the absence of EGF and also significantly potentiated the cell growth in response to EGF treatment in a dose-dependent manner (Figure 2B. Elevated expression of Vav3 upon transient transfection of Vav3 expression vector was confirmed by western blot analysis in these cells (Figure 2C).

We then determined whether knockdown expression of Vav3 inhibits growth of these breast cancer cells using Vav3 siRNA that has been characterized previously [25]. Lipofectamine 2000 used for transfection of siRNA has been shown to knock down more than $80 \%$ activity of the endogenous gene in a panel of cells tested (Invitrogen). We confirmed a reduced level of Vav3 upon transfection of Vav3 siRNA by protein expression analysis relative to the control (Figure 2D). A growth stimulatory effect in response to estrogen stimulation was observed in T47D and MCF7 cells (Figure 2Eand 2F). We found that knockdown expression of Vav3 significantly inhibited both estrogen-dependent and -independent growth in these breast cancer cells (Figure 2Eand 2F). At 1.2 pM of siRNA, the growth of T47D and MCF7 cells was inhibited by $64 \%$ and $68 \%$ in the absence of estrogen and $46 \%$ and $77 \%$ in the presence of estrogen relative to their respective controls. These data suggest that Vav3 is involved in both estrogen-dependent and -independent growth in breast cancer cells. 
A.

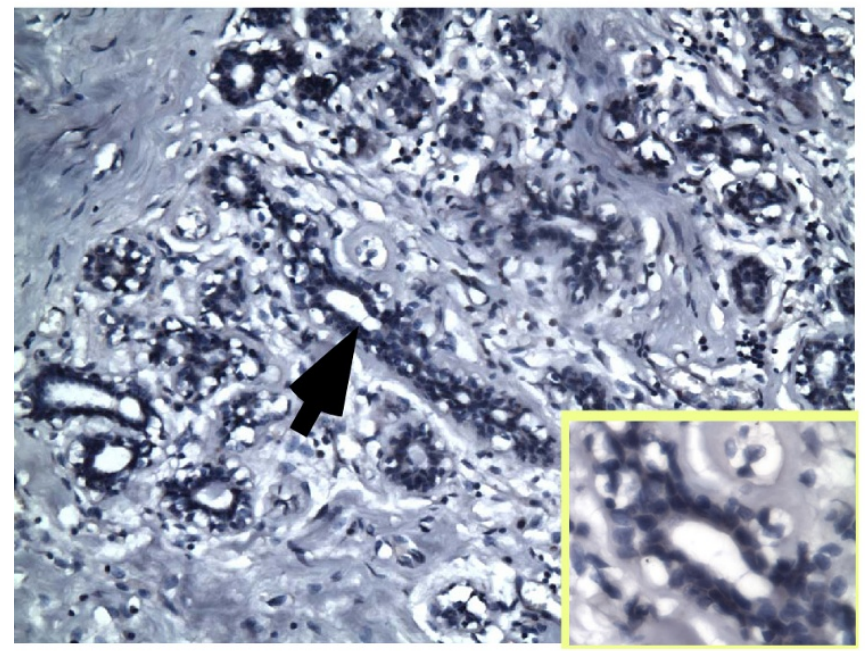

C.

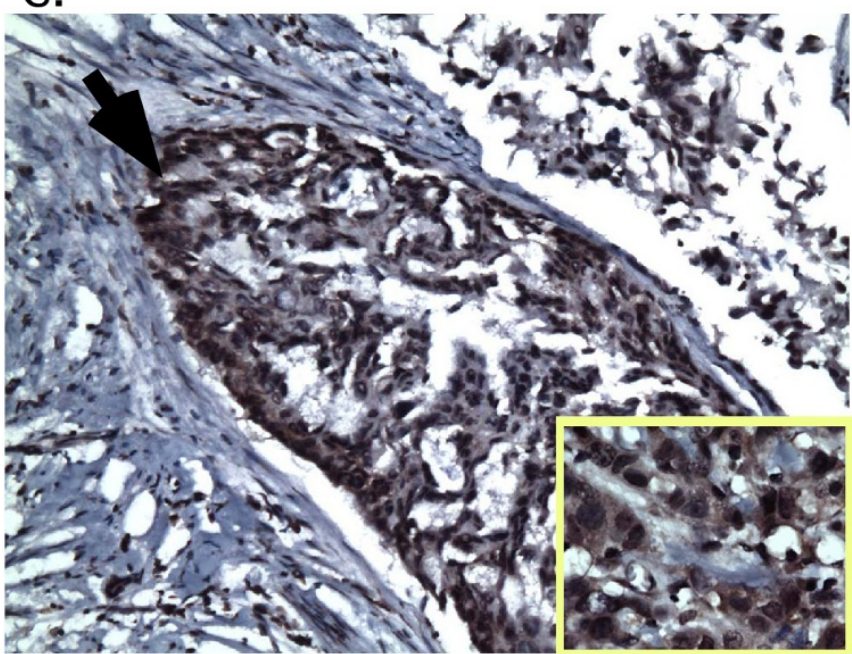

B.

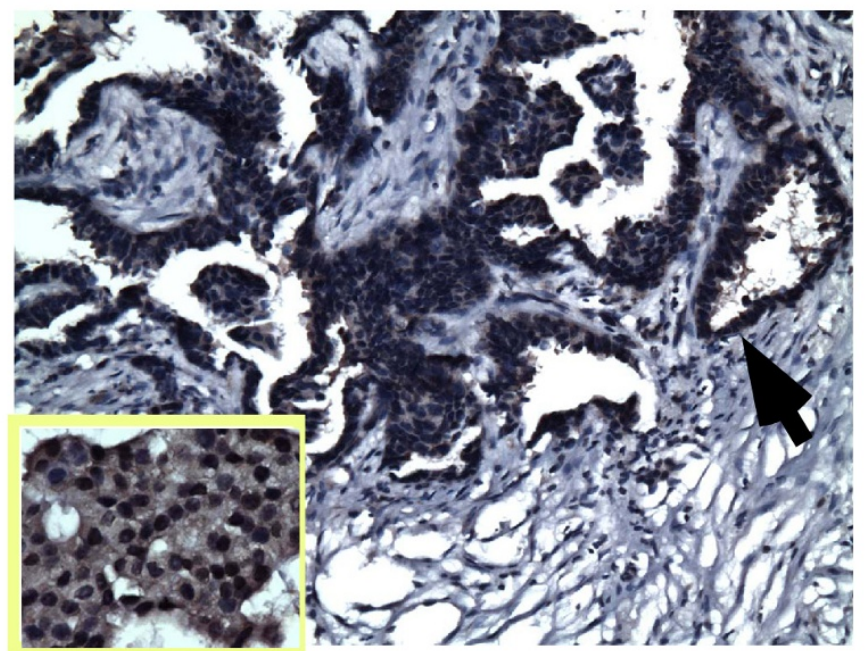

D.

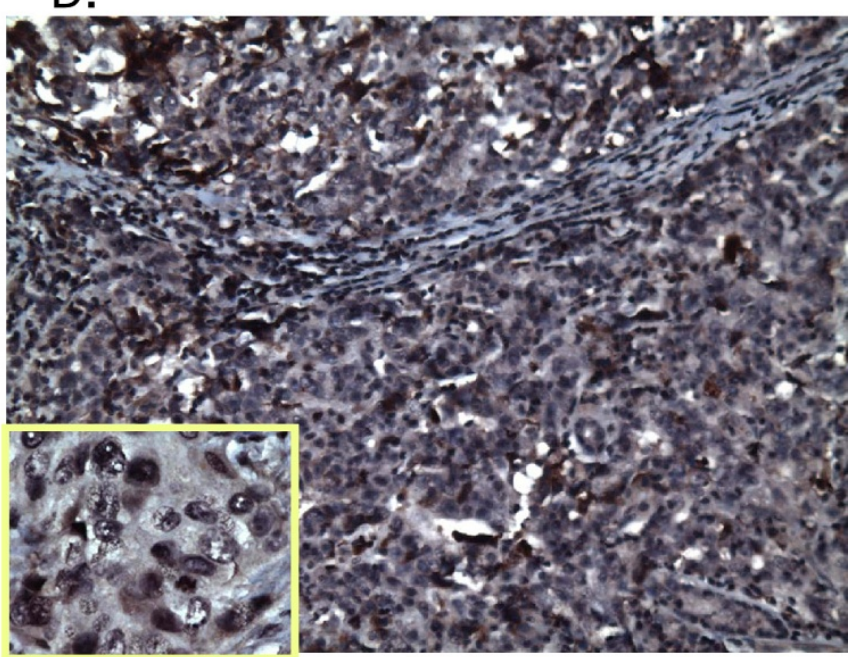

Figure I

Overexpression of Vav3 in human breast cancer. (A) The normal breast epithelial cells reveal negative immunoreactivity for Vav3 (200x magnification). Breast adenocarcinomas with well-differentiation (B), moderately differentiation(C), and poorly differentiation (D) show positive immunoreactivity (brown staining) for Vav3 in both nucleus and cytoplasm. Arrows indicate the breast epithelial and cancer cells. The microphotographs indicate nucleus feature of the breast epithelial and breast cancer cells (400x magnification). For moderately and poorly differentiation breast cancer cells, the nuclei are significantly enlarged and hyperchromatic with coarse clumping of chromatin, prominent nucleoli, and irregular nuclear membrane.

\section{Vav3 upregulates ER $\alpha$ activity}

Previous studies have revealed that Vav3*, a Vav3 mutant with N-terminal domain deletion including the $\mathrm{AD}$ domain containing three tyrosine residues, is a constitutive active form and has much stronger oncogenic effect relative to that by wild type Vav3 [30]. Structure analysis of Vav protein suggested that in the non-phosphorylation state, Vav protein is folded, which is achieved by binding of tyrosines in the $\mathrm{AD}$ domain to the $\mathrm{DH}$ domain and binding of the $\mathrm{CH}$ domain to the $\mathrm{C} 1$ region [6]. Phosphorylation of the tyrosines in the $\mathrm{AD}$ domain results in unfolding of Vav protein and exposure of the DH domain for interacting with substrate proteins. Consistently, we found that the constitutive active Vav3* shows a much stronger activity in upregulation of AR activity than that by Vav3 [25].

To determine whether Vav3 regulates ER $\alpha$ activity, we used both Vav3 and Vav3* for the reporter assay. Hela cells were transiently cotransfected with a luciferase reporter driven by the estrogen response element (ERE), ER $\alpha$ expression vector, and either Vav3 or Vav3* expres- 
A.

C.

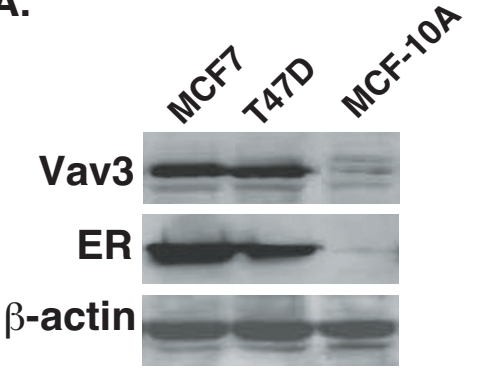

B.

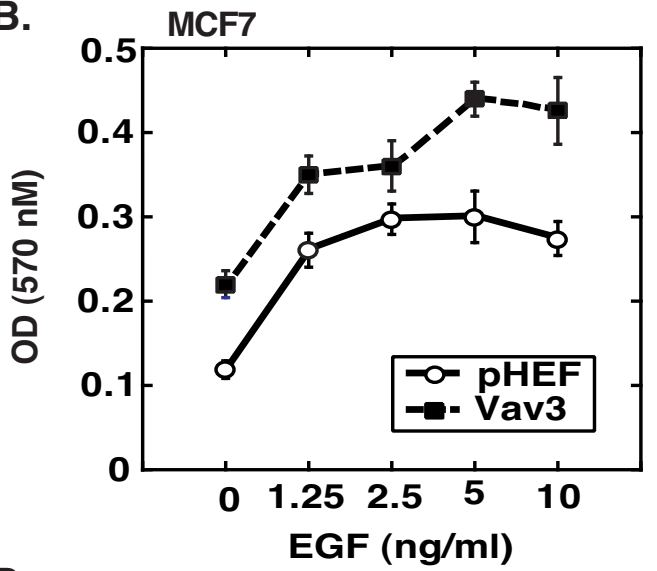

D.

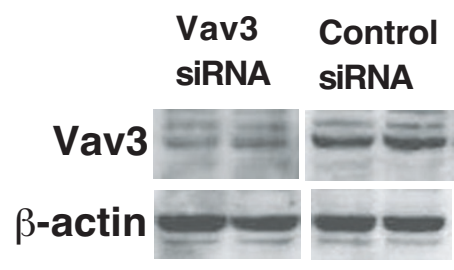

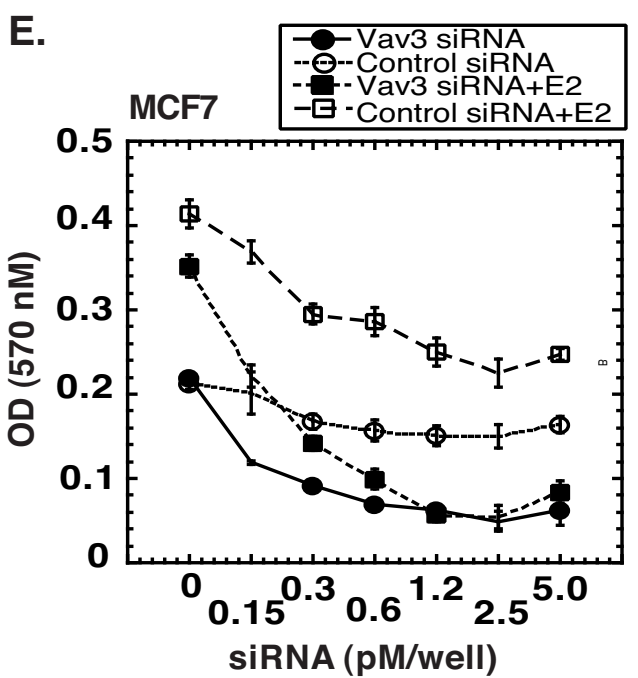

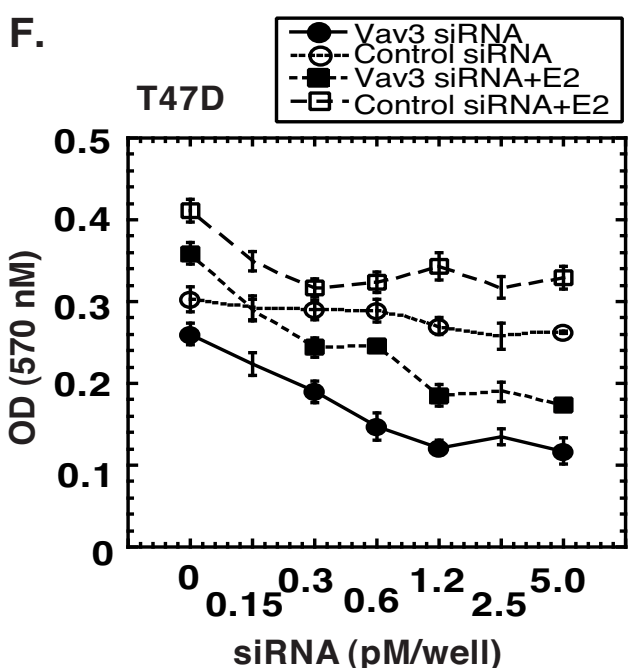

Figure 2

Vav3 is involved in growth of breast cancer cells. (A) Expression analysis of Vav3 and ER $\alpha$ in breast cancer MCF7 and T47D cells and nontumoral breast epithelial MCF-IOA cells. The cell extracts were prepared from T47D, MCF7, and MCF-I0A cells and subjected to western blot analysis for Vav3 and ER $\alpha$. $\beta$-actin was served as loading control. (B) MCF7 cells were transiently transfected with Vav3 expression vector or control empty vector and then cultured in stripped medium in the absence or presence of EGF for 5 days, followed by MTT assay. The data was presented as absorbance at OD 570 nM. (C) MCF7 cells were transiently transfected with Vav3 expression vector or empty pHEF vector for 3 days, followed by cell extracts preparation and western blot analysis for Vav3. $\beta$-actin was served as loading control. (D) Knock down expression of Vav3 upon transfection of Vav3 siRNA. T47D cells were transfected with 5 pM/well of siVav3-247 or control-247 in 6-well plate for 3 days. The cell extracts were prepared and subjected to western blot analysis for Vav3. $\beta$-actin was served as loading control. (E and $F$ ) MCF7 cells and T47D cells ( 2500 cells/well in 96-well plate) were transiently transfected with siVav3-247 or control-247 at the concentrations of $0,0.15,0.3,0.6,1.2,2.5,5.0 \mathrm{pM} /$ well using Lipofectamine 2000 for overnight. Then, the cells were cultured in stripped medium without or with E2 (10-9 M) for 5 days, followed by MTT assay. The data was presented as absorbance at OD $570 \mathrm{nM}$. 
A.
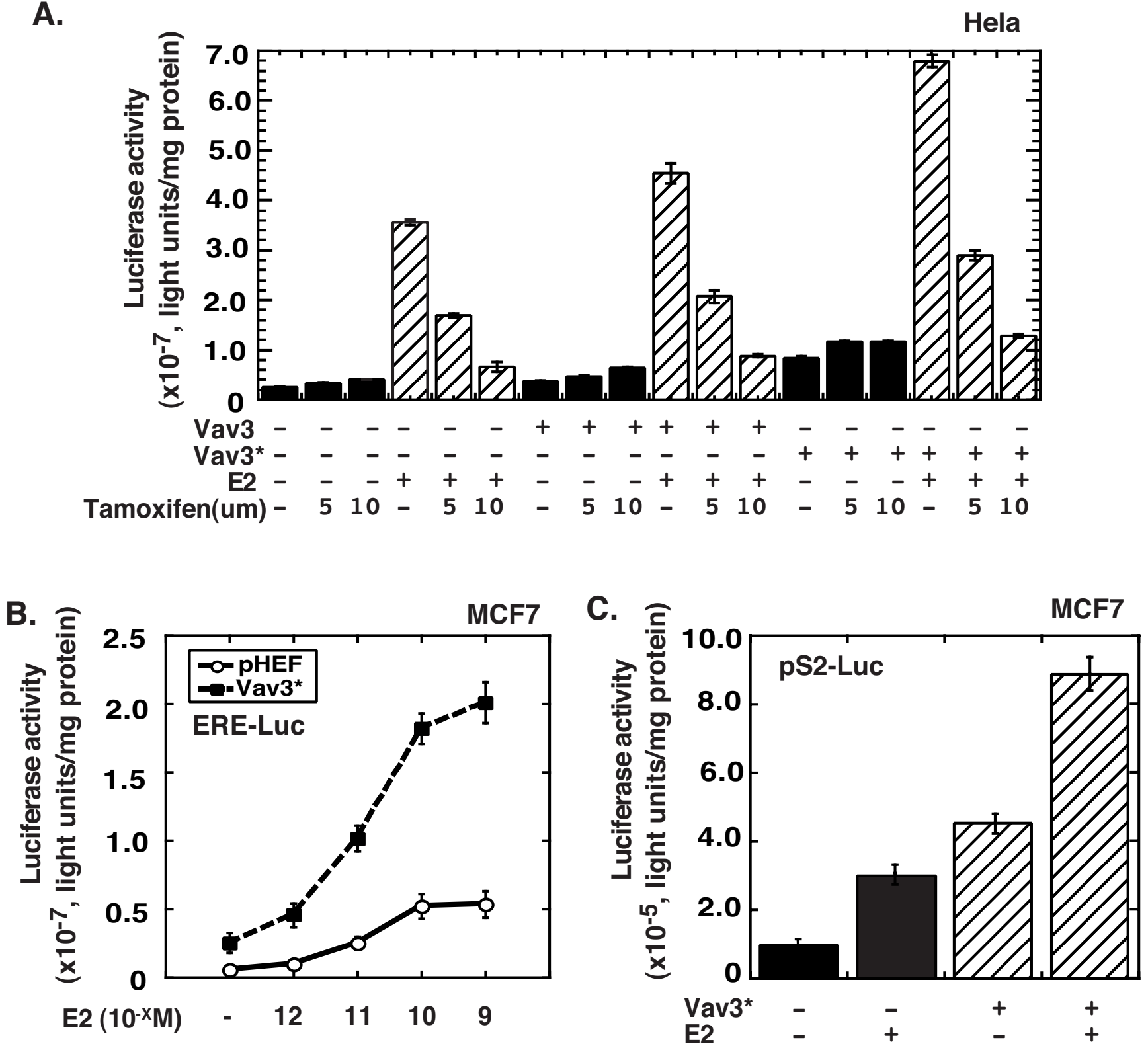

Figure 3

Vav3 enhances ER $\alpha$ activity. (A) Hela cells ( $10^{5}$ cells/well in I2-well plate) were cotransfected with ERE-Luc (0.5 ug), expression vectors for Vav3, Vav3*, or empty vector pHEF (200 ng) and ER $\alpha(50 \mathrm{ng})$, respectively. Then, the cells were treated without or with E2 (I0-9 M) and without or with Tamoxifen. (B and C) MCF7 cells ( $10^{5}$ cells/well in I2-well plate) were cotransfected with ERE-Luc (0.5 ug) (B) or PS2-Luc (0.5 ug) (C), and expression vector (0.25 ug) for Vav3*, or empty vector $\mathrm{PHEF}$, respectively. Then, the cells were treated with E2. All transfection and drug treatment are in stripped medium for 24 hours, followed by luciferase assay. Renilla luciferase as an internal control was used to normalize the data. Data are presented as the mean $( \pm S D)$ of duplicate values of a representative experiment that was independently repeated for five times.

sion vector. Overexpression of Vav3, and Vav3* with a greater effect relative to the empty vector $\mathrm{pHEF}$, enhanced both basal and estrogen-stimulated ER $\alpha$ activity (Figure 3A). Furthermore, we found that both estrogen-stimulated and Vav3-activated ER $\alpha$ activities were blocked by ER antagonist Tamoxifen (Figure 3A). The inhibitory effect for ER $\alpha$ by Tamoxifen was compromised with Vav3 overexpression. Upregulation of the endogenous ER $\alpha$ activity by Vav3 relative to the empty vector $\mathrm{pHEF}$ was also confirmed in MCF7 cells by stimulation of luciferase reporter expression driven by ERE (Figure 3B) and natural promoter of ER $\alpha$ target gene pS2 (Figure 3C). Vav3 also 
significantly enhanced ER $\alpha$ activity stimulated by subphysiological concentrations of estrogen $\left(10^{-11}\right.$ and $10^{-10}$ $\mathrm{M})$ (Figure 3B). These data suggest that Vav3 overexpression confers ER $\alpha$ hypersensitivity.

We next examined the domains of Vav3 required for upregulation of ER $\alpha$ activity by analyzing both wild type Vav3 and Vav3 deletion mutants (Figure 4A). We found that Vav3* demonstrated a much stronger activity for ER $\alpha$ activation relative to Vav3 as compared with the empty vector $\mathrm{pHEF}$, supporting the previous observation that activity of Vav3 is subjected to regulation by phosphorylation (Figure 4Band Figure 5D) [6]. In addition, deletion of the DH domain, but not the $\mathrm{SH}$ domain or the $\mathrm{CH}$ domain, abolished Vav3 function for ER $\alpha$ activation (Figure $4 \mathrm{~B}$ ). The truncated Vav3 protein with deletion of both $\mathrm{PH}$ and $\mathrm{SH}$ domains failed to activate ER $\alpha$. Therefore, consistent with our previous observation of its effect for
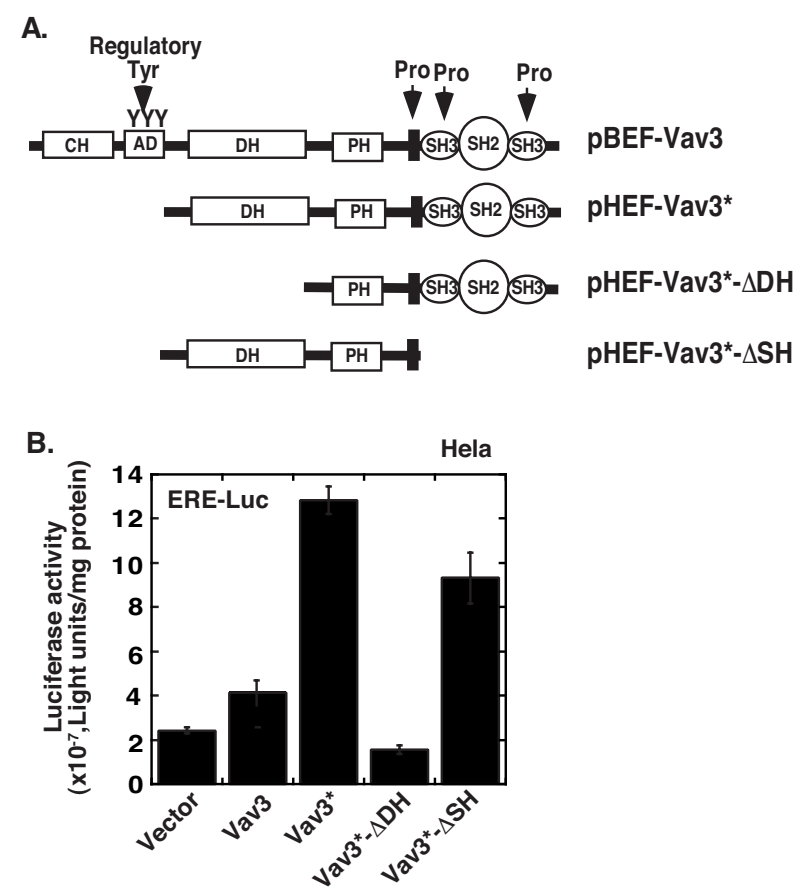

Figure 4

Determination of the functional domain of Vav3 involved in ER $\alpha$ activation. (A) Deletion constructs of Vav3. (B) Hela cells ( $10^{5}$ cells/well in 12-well plate) were cotransfected with ERE-Luc (0.5 ug) and expression vectors (200 ng) for Vav3, Vav3*, Vav3*- $\Delta \mathrm{DH}, \mathrm{Vav3}{ }^{*}-\Delta \mathrm{SH}$, or empty vector $\mathrm{PHEF}$, as well as expression vector $(50 \mathrm{ng})$ for $\mathrm{ER} \alpha$, respectively. All transfection and drug treatment are in stripped medium for 24 hours, followed by luciferase assay. Renilla luciferase as an internal control was used to normalize the data. Data are presented as the mean $( \pm S D)$ of duplicate values of a representative experiment that was independently repeated for five times.
$\mathrm{AR}$, the DH domain of Vav3 is also essential for ER $\alpha$ activation [25].

\section{Vav3 enhances ER $\alpha$ activity partially via the PI3K-Akt pathway and potentiates EGF for ER $\alpha$ activation}

Elevated PI3K-Akt signaling enhances ER $\alpha$ activity and is thought to be critical for breast cancer development and progression [11,31,32]. Since Vav3 is a potential upstream regulator of the PI3K-Akt pathway $[33,34]$, we investigated whether Vav3 overexpression impacts on PI3K-Akt signaling-mediated ER $\alpha$ activation. Hela cells were transiently cotransfected with ERE-Luc reporter, and expression vectors $\mathrm{ER} \alpha, \mathrm{Vav} 3 *$, and $\mathrm{p} 85$ (the regulatory subunit of PI3K) and p110 (the catalytic subunit of PI3K). As shown in Figure 5A, cotransfection of Vav3* or PI3K relative to the empty vector $\mathrm{pHEF}$ or pCR3.1 stimulated ER $\alpha$ activity. ER $\alpha$ activity was further enhanced in cells cotransfected with both Vav3* and PI3K. On the other hand, cotransfection of a dominant-negative Akt relative to the empty vector pCR3.1 blocked Vav3*-mediated ER $\alpha$ activation (Figure 5B). Next, we determined the role of endogenous PI3K in Vav3-induced ER $\alpha$ activation. Data in Figure 5C showed that PI3K inhibitor Wortmannin blocked both basal and Vav3-stimulated ER $\alpha$ activation in the absence and presence of E2. Similar results were also noted in cells treated with PI3K inhibitor LY294002 (data not shown). These data indicate that Vav3 activates ER $\alpha$ at least partially via PI3K-Akt signaling.

In agreement with its effects on estrogen-independent growth in breast cancer cells (Figure 2B), overexpression of Vav3 potentiated EGF-stimulated ER $\alpha$ activation (Figure 5D). This data suggests that Vav3 overexpression may mediate EGFR/HER2/neu-elicited signaling leading to ER $\alpha$ hypersensitivity.

\section{Vav3 complexes with ER $\alpha$ and impacts on ER $\alpha$ signaling axis}

Recent findings implicate that Vav family proteins also complex with transcription factors and regulate gene expression [35,36]. A nuclear localization signal (NLS) in the $\mathrm{PH}$ domain was shown to be solely responsible for the nucleus localization of Vav1 protein, indicating a role of Vav family protein as a transcription coregulator. We have demonstrated that the DH domain of Vav3 is essential for $\mathrm{AR}$ and ER $\alpha$ activation (Figure 4) [25]. We performed sequence analysis of Vav proteins and found that the $\mathrm{DH}$ domain of Vav3 contains three consensus sequences of the LXXLL motifs or NR boxes, which have been well characterized and involved in interaction with nuclear receptors (Figure 6A). In addition, homologous analysis of Vav3 and Vav1 genes identified a conserved NLS in the PH domain of Vav3 (Figure 6B). These findings suggest that Vav3 is also localized in nucleus and regulates transcription of nuclear receptors. 

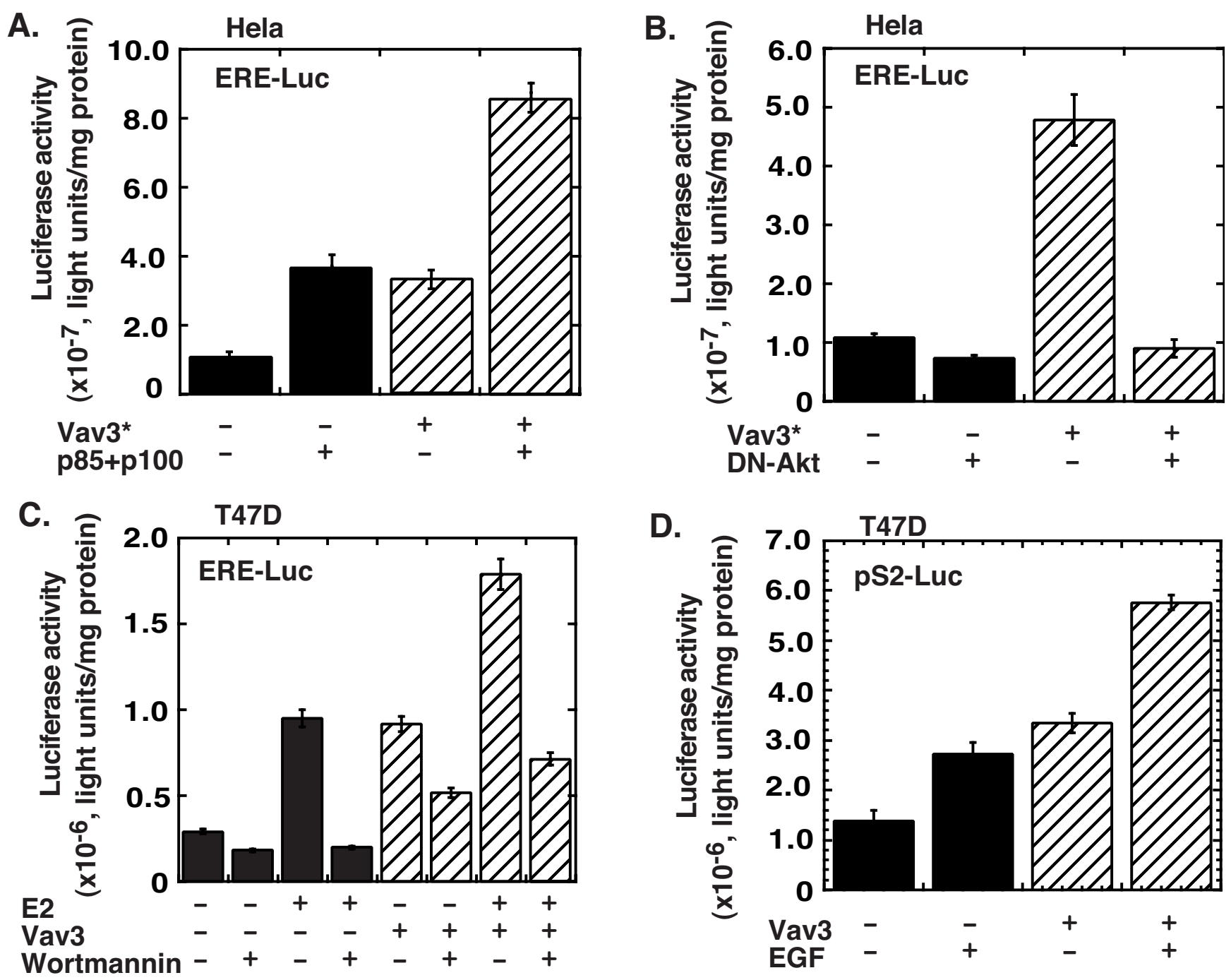

\section{Figure 5}

Vav3 activates ER $\alpha$ partially via PI3K-Akt signaling and potentiates EGF for ER $\alpha$ activation. (A) Hela cells were cotransfected with ERE-Luc (0.25 ug), expression vectors ER $\alpha$ ( $25 \mathrm{ng})$, Vav3* or empty vector pHEF (50 ng), p85+p I I0 or empty vector pCR3.I (50 ng). (B) Hela cells were cotransfected with ERE-Luc reporter (0.25 ug/well in I2-well plate), expression vectors $\mathrm{ER} \alpha(25 \mathrm{ng})$, dominant-negative Akt expression vector or empty vector pCR3.I (0.I ug), and Vav3* expression vector or empty vector pHEF (0.I ug), respectively. (C) T47D cells were cotransfected with ERE-Luc (0.5 ug) and expression vector for Vav3 or empty vector pHEF $(0.25 \mathrm{ug})$. Then, the cells were treated with Wortmannin (0.5 um). (D) T47D cells were cotransfected with pS2-Luc (0.5 ug) and expression vector for Vav3 or empty vector pHEF ( 0.25 ug). Then, the cells were treated with EGF $(20 \mathrm{ng} / \mathrm{ml})$. All transfection and drug treatment are in stripped medium for 24 hours, followed by luciferase assay. Renilla luciferase as an internal control was used to normalize the data. Data are presented as the mean ( \pm SD) of duplicate values of a representative experiment that was independently repeated for five times.

We then determined whether Vav3 complexes with ER $\alpha$. We performed GST pull down experiment to confirm the interaction between Vav3 and ER $\alpha$. A GST fusion protein including the DH and PH domain of Vav3 (GST-Vav3$\mathrm{DH}+\mathrm{PH}$ ) was generated (Figure $7 \mathrm{~A}$ ). Cell extract derived from MCF7 cells was incubated with immobilized GSTVav3-DH+PH fusion protein or GST protein. Then, the pull down samples were fractionated by SDS-PAGE (Figure 7B) and subjected to western blot analysis for ER $\alpha$. We found that GST-Vav3-DH+PH fusion protein, but GST protein, interacted with $\mathrm{ER} \alpha$ (Figure 7C). In summary, we found that Vav3 contains NLS in the PH domain and three LXXLL motifs in the DH domain. The deletion mutation and functional analysis by luciferase reporter assay 


\section{A. $\quad \frac{I}{* \star} \quad \frac{\text { II }}{* \star} \frac{\text { III }}{\star *}$ \\ Vav3 304 KLEECSKRANNGKFTLRDLLVVPMQRVLKYHLLLQELVKHTTDPTEKANLKLALD KLEECS+RANNG+FTLRDLL+VPMQRVLKYHLLLQELVKHT++++EK+NL+LALD \\ Vav1 306 KLEECSQRANNGRFTLRDLLMVPMQRVLKYHLLLQELVKHTQEAMEKENLRLALD}

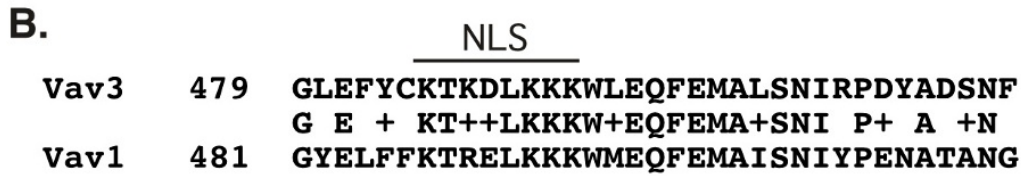

\section{Figure 6}

Sequence analysis of Vav3 and VavI genes. (A) The consensus sequences of LXXLL motifs I, II, and III were identified in the DH domain of Vav3 as indicated. Mutation of LLLQELV sequence overlapping with LXXLL motifs II and III has been shown to abolish the GEF activity of Vav3. (B) A consensus sequence of the nucleus localization signal (NLS) in Vav3 was localized in the $\mathrm{PH}$ domain.

showed that the DH domain of Vav3 is essential for enhancing ER $\alpha$ activity and is involved in complex with ER $\alpha$. Our data suggest that Vav3 complexes with ER $\alpha$ and its overexpression enhances ER $\alpha$ signaling axis in breast cancer cells.

A.

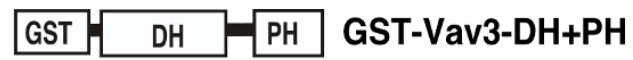

B.

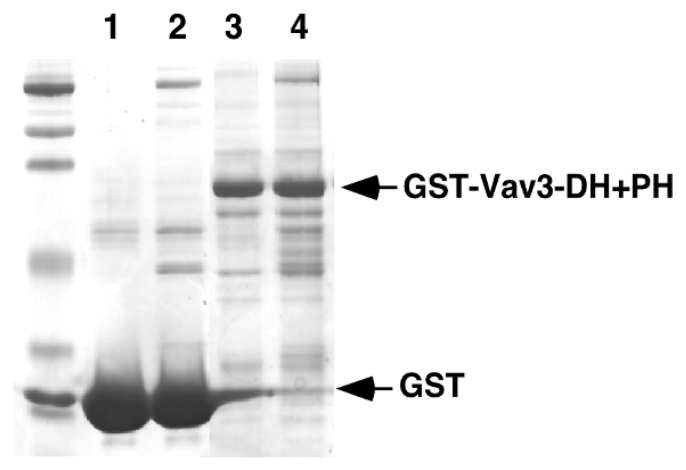

C.

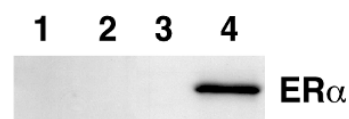

\section{Figure 7}

Vav3 complexes with ER $\alpha$ by GST pull down analysis. (A) GST-Vav3-DH+PH fusion protein. (B) GST-Vav3$\mathrm{DH}+\mathrm{PH}$ fusion protein (lane 3 and 4 ) and control GST protein (lane I and 2) were subjected to pull down reaction in the absence (lane I and 3 ) and presence (land 2 and 4 ) of cell extract derived from MCF7 cells. The samples were fractionated in SDS-PAGE and stained with Coomassie Blue. (C) The same samples were subjected to western blot analysis for $\mathrm{ER} \alpha$.

\section{Discussion}

Previous studies from our group have demonstrated that Vav3 is overexpressed in human prostate cancer and potentiates AR signaling [25]. Breast cancer and prostate cancer are steroid-dependent tumors and share a significant similarity in their characteristics and treatment. For instance, growth of these cancer cells, mediated by their corresponding hormone receptors $\mathrm{ER} \alpha$ and $\mathrm{AR}$, is hormone-dependent. Hormone ablation is common therapy for both cancers. Recurrent diseases develop hormoneindependent growth. Given that steroidal nuclear receptors share many common properties, we hypothesized that Vav3 may regulate $\mathrm{ER} \alpha$ activity and is involved in human breast cancer. We tested this hypothesis in the present study by examining the expression of Vav3 in human breast cancer specimens and cell lines and investigated a potential role of Vav3 in breast cancer cell growth and ER $\alpha$ signaling. We found that Vav3 was overexpressed in human breast cancer, particularly in the poorly differentiated lesions and in the two most commonly used breast cancer cell lines. The knockdown expression of Vav3 compromised both estrogen-stimulated and -independent growth of breast cancer cells. On the other hand, overexpression of Vav3 enhanced ER $\alpha$ signaling. These data strongly suggest that Vav3 may play an important role in breast cancer development and/or progression.

Vav3 is an oncogene identified in cell transformation experiments [3]. Vav3 is activated upon ligand stimulation of EGF, insulin, Ros, and IGF receptors and physically associates with a variety of signaling molecules, including Rac1, Cdc42, PI3K, Grb2, and PLC- $\gamma$, leading to alteration in cell morphology and cell transformation [33]. Overexpression of Vav3 leads to PI3K activation and focus formation in NIH3T3 cells [34]. In contrast, blocking PI3K 
activation by PTEN and LY294002 inhibits Vav3-induced cell transformation. Furthermore, it has been shown that Vav3*, a Vav3 mutant with N-terminal domain deletion including the acidic domain, is a constitutive active form and has much stronger oncogenic effect compared with that by Vav3 [30]. Consistently, we found that the augmentation of ER $\alpha$ signaling by Vav3 overexpression was similarly inhibited by Wortmannin and by overexpression of a dominant-negative Akt. In addition, Vav3 showed a lower activity for ER $\alpha$ activation relative to that by Vav3*. EGF treatment significantly potentiated Vav3 activity for ER $\alpha$ activation. These data suggest that Vav3 is subjected to regulation by phosphorylation, most likely at the three tyrosines in the AD domain, which may cause conformation change, release the inhibitory effect of the N-terminal domain, and expose the DH domain for ER $\alpha$ binding.

Many coactivators of steroid hormone receptors have the LXXLL motifs, where $\mathrm{L}$ is leucine and $\mathrm{X}$ is any amino acid $[37,38]$. These coregulators interact with and upregulate ER $\alpha$-mediated signaling in both nucleus and cytoplasm. For instance, SRC-1 and its related proteins are a family of coactivators containing the homologous bHLH-PAS domain and receptor-interacting domain (RID) with multiple LXXLL motifs and enhance transcription activity of nuclear receptors $[37,38]$. PELP1/MNAR containing the LXXLL motif interacts with and enhances both genomic and nongenomic ER $\alpha$ activities [21,24]. Recent findings implicate that Vav family proteins also complex with transcription factors and regulate gene expression. Vav1 was identified in the component of transcriptionally active nuclear factor of activated T cells (NFAT)- and nuclear factor NFkB-like complexes [35,36]. A nuclear localization signal (NLS) in the PH domain is solely responsible for nucleus localization of Vav1 protein, indicating a role of Vav family proteins as a transcription coregulator. We found that Vav3 contains the LXXLL motifs in the DH domain and NLS in the PH domain. This finding suggests that Vav3 is also a nuclear protein.

Previous study has shown that Vav protein can be activated by receptor tyrosine kinase upon activation of EGFR [39]. Furthermore, ER $\alpha$ resides in multi-protein complexes with molecules, such as MNAR/PELP1 and src, in the cytoplasm and signals through the PI3K-Akt and MAPK pathways in breast cancer cells [23,24]. ER $\alpha$ was also found localized in lipid rafts and involved in signaling elicited by EGFR and HER2 receptors [40,41]. We found that Vav3 activates ER $\alpha$ partially via PI3K-Akt signaling and potentiates EGF effect for cell growth and ER $\alpha$ activation in breast cancer cells. More interestingly, we found that Vav3 complexes with ER $\alpha$. These findings suggest that Vav3 enhances ER $\alpha$ signaling axis in breast cancer cells. Vav3 overexpression may confer ER $\alpha$ hypersensitivity and play a role in breast cancer. Given both nuclear and cytoplasmic localization of Vav3 protein, our data implicate that Vav3 may impact on both genomic and nongenomic ER $\alpha$ activity. Furthermore, the relationship of Vav3 and ER $\alpha$ in the context of EGFR/ HER2 and PI3K-Akt signaling is remained to be determined.

Our findings support the notion that Vav3 overexpression may play a role in breast cancer, based on the following reasons: 1) Vav3 is overexpressed and correlated with poorly differentiated tumors in human breast cancer; 2) Vav3 contains the LXXLL motifs and complexes with ER $\alpha$; 3) Vav3 enhances ER $\alpha$ activity partially via the PI3K-Akt pathway; 4) Vav3 is a protein with multiple domains and functions, including the $\mathrm{SH} 2$ domain interacting with receptor protein tyrosine kinase, the $\mathrm{PH}$ domain binding PIP3 involved in association with the cell membrane, and the DH domain involved in interaction with $\mathrm{ER} \alpha$; 5) Vav3 potentiates EGF for cell growth and ER $\alpha$ activation. Taken together, these findings suggest that Vav3 overexpression enhances ER $\alpha$-mediated signaling axis and may be involved in breast cancer.

Data presented in this report clearly show that Vav3 is overexpressed in human breast cancer and is involved in growth of breast cancer cells and ER $\alpha$ signaling. Whereas our data showed that Vav3 complexes with ER $\alpha$, molecular mechanisms underlying the enhancement of ER $\alpha$ signaling remain to be elucidated. Nevertheless, data presented here strongly suggest a novel mechanism that potentially leads to ER $\alpha$ hypersensitivity and breast cancer development and/or progression.

\section{Abbreviations}

ER $\alpha$ : estrogen receptor $\alpha$; EGFR: epidermal growth factor receptor; HER2: human epidermal growth factor receptor; GEF: quanine nucleotide exchange factor; IHC: Immunohistochemistry; NLS: nuclear localization signal.

\section{Competing interests}

The authors declare that they have no competing interests.

\section{Authors' contributions}

KL generated GST fusion protein for pull down analysis. YL performed cell cultures, proliferation assays, Western blot analysis, and reporter assay. ZD performed IHC. JQM did histopathological analysis of breast cancer tissues. JZ provided plasmid and expertise in protein/protein interaction. SL contributed to conception and design of study and interpretation of data. All authors read and approved the final manuscript.

\section{Acknowledgements}

This work is supported by grants from the American Cancer Society Ohio Division, Inc., Ohio Cancer Research Associates, and NIH ROICAII 9935. 


\section{References}

I. Bustelo XR: Regulatory and signaling properties of the Vav family. Mol Cell Biol 2000, 20(5):|46|-|477.

2. Bustelo $X R:$ Vav proteins, adaptors and cell signaling. Oncogene 200I, 20(44):6372-638I.

3. Katzav S, Martin-Zanca D, Barbacid M: vav, a novel human oncogene derived from a locus ubiquitously expressed in hematopoietic cells. Embo J 1989, 8(8):2283-2290.

4. Zugaza JL, Lopez-Lago MA, Caloca MJ, Dosil M, Movilla N, Bustelo XR: Structural determinants for the biological activity of Vav proteins. J Biol Chem 2002, 277(47):45377-45392.

5. Moores SL, Selfors LM, Fredericks J, Breit T, Fujikawa K, Alt FW, Brugge JS, Swat W: Vav family proteins couple to diverse cell surface receptors. Mol Cell Biol 2000, 20(I 7):6364-6373.

6. Aghazadeh B, Lowry WE, Huang XY, Rosen MK: Structural basis for relief of autoinhibition of the Dbl homology domain of proto-oncogene Vav by tyrosine phosphorylation. Cell 2000, I 02(5):625-633.

7. Sommer S, Fuqua SA: Estrogen receptor and breast cancer. Semin Cancer Biol 200I, I I(5):339-352.

8. Hennessy BT, Smith DL, Ram PT, Lu Y, Mills GB: Exploiting the PI3K/AKT pathway for cancer drug discovery. Nature reviews 2005, 4( I 2):988-1004.

9. Rau KM, Kang HY, Cha TL, Miller SA, Hung MC: The mechanisms and managements of hormone-therapy resistance in breast and prostate cancers. Endocrine-related cancer 2005, | 2(3):5 | |-532.

10. Glaeser M, Floetotto T, Hanstein B, Beckmann MW, Niederacher D: Gene amplification and expression of the steroid receptor coactivator SRC3 (AIB I) in sporadic breast and endometrial carcinomas. Horm Metab Res 200I, 33(3): I2I-I 26.

II. Rochette-Egly C: Nuclear receptors: integration of multiple signalling pathways through phosphorylation. Cell Signal 2003, I 5(4):355-366.

12. Mills GB, Lu Y, Fang X, Wang H, Eder A, Mao M, Swaby R, Cheng KW, Stokoe $D$, Siminovitch $K$, Jaffe $R$, Gray J: The role of genetic abnormalities of PTEN and the phosphatidylinositol 3-kinase pathway in breast and ovarian tumorigenesis, prognosis, and therapy. Semin Oncol 200I, 28(5 Suppl I6): I25-|4|

13. Isakoff SJ, Engelman JA, Irie HY, Luo J, Brachmann SM, Pearline RV, Cantley LC, Brugge JS: Breast cancer-associated PIK3CA mutations are oncogenic in mammary epithelial cells. Cancer research 2005, 65(23): $10992-11000$.

14. Li SY, Rong M, Grieu F, lacopetta B: PIK3CA mutations in breast cancer are associated with poor outcome. Breast Cancer Res Treat 2005: I-5

15. Clark AS, West K, Streicher S, Dennis PA: Constitutive and inducible Akt activity promotes resistance to chemotherapy, trastuzumab, or tamoxifen in breast cancer cells. Mol Cancer Ther 2002, I(9):707-7I7.

16. Ali S, Coombes RC: Endocrine-responsive breast cancer and strategies for combating resistance. Nat Rev Cancer 2002, 2(2): $101-112$.

17. Felekkis KN, Narsimhan RP, Near R, Castro AF, Zheng Y, Quilliam LA, Lerner A: AND-34 activates phosphatidylinositol 3-kinase and induces anti-estrogen resistance in a SH2 and GDP exchange factor-like domain-dependent manner. Mol Cancer Res 2005, 3(I):32-4I.

18. Guy CT, Webster MA, Schaller M, Parsons TJ, Cardiff RD, Muller WJ: Expression of the neu protooncogene in the mammary epithelium of transgenic mice induces metastatic disease. Proceedings of the National Academy of Sciences of the United States of America 1992, 89(22): 10578-10582

19. Blanco-Aparicio C, Perez-Gallego L, Pequeno B, Leal JF, Renner O, Carnero A: Mice expressing myrAKTI in the mammary gland develop carcinogen-induced ER-positive mammary tumors that mimic human breast cancer. Carcinogenesis 2007, 28(3):584-594.

20. Maroulakou IG, Oemler W, Naber SP, Tsichlis PN: Akt I ablation inhibits, whereas Akt2 ablation accelerates, the development of mammary adenocarcinomas in mouse mammary tumor virus (MMTV)-ErbB2/neu and MMTV-polyoma middle T transgenic mice. Cancer research 2007, 67(I): 167-177.

21. Vadlamudi RK, Kumar R: Functional and biological properties of the nuclear receptor coregulator PELPI/MNAR. Nuclear receptor signaling 2007, 5:e004.
22. Gururaj AE, Rayala SK, Vadlamudi RK, Kumar R: Novel mechanisms of resistance to endocrine therapy: genomic and nongenomic considerations. Clin Cancer Res 2006, I2(3 Pt 2): $1001 \mathrm{~s}-1007 \mathrm{~s}$

23. Greger JG, Fursov N, Cooch N, McLarney S, Freedman LP, Edwards DP, Cheskis B]: Phosphorylation of MNAR promotes estrogen activation of phosphatidylinositol 3-kinase. Mol Cell Biol 2007. 27(5): 1904-1913.

24. Wong CW, McNally C, Nickbarg E, Komm BS, Cheskis BJ: Estrogen receptor-interacting protein that modulates its nongenomic activity-crosstalk with Src/Erk phosphorylation cascade. Proceedings of the National Academy of Sciences of the United States of America 2002, 99(23): | 4783-14788.

25. Dong ZY, Liu Y, Lu S, Wang A, Lee K, Wang LH, Revelo M, Lu S: Vav3 oncogene is overexpressed and regulates cell growth and androgen receptor activity in human prostate cancer. Mol Endo 2006, 20:2315-2325.

26. Lyons LS, Burnstein KL: Vav3, a Rho GTPase Guanine Nucleotide Exchange Factor, Increases During Progression to Androgen Independence in Prostate Cancer Cells and Potentiates Androgen Receptor Transcriptional Activity. Mol Endocrinol 2005. December 29

27. Lu S, Tsai SY, Tsai MJ: Melecular mechanisms of androgen-independent growth of human prostate cancer LNCaP-Al cells. Endocrinology 1999, I40:5054-5059.

28. Zhang J, Kalkum M, Yamamura S, Chait BT, Roeder RG: E protein silencing by the leukemogenic AMLI-ETO fusion protein. Science 2004, 305(5688): I 286-1289.

29. Zhang J, Kalkum M, Chait BT, Roeder RG: The N-CoR-HDAC3 nuclear receptor corepressor complex inhibits the JNK pathway through the integral subunit GPS2. Mol Cell 2002, 9(3):6 I |-623.

30. Lopez-Lago M, Lee H, Cruz C, Movilla N, Bustelo XR: Tyrosine phosphorylation mediates both activation and downmodulation of the biological activity of Vav. Mol Cell Biol 2000, 20(5): |678-|69|.

31. Tremblay A, Tremblay GB, Labrie F, Giguere V: Ligand-independent recruitment of SRC-I to estrogen receptor beta through phosphorylation of activation function AF-I. Mol Cell 1999, 3(4):5।3-519.

32. Yeh S, Lin HK, Kang HY, Thin TH, Lin MF, Chang C: From HER2/ Neu signal cascade to androgen receptor and its coactivators: a novel pathway by induction of androgen target genes through MAP kinase in prostate cancer cells. Proceedings of the National Academy of Sciences of the United States of America 1999. 96:5458-5463.

33. Zeng L, Sachdev P, Yan L, Chan JL, Trenkle T, McClelland M, Welsh J, Wang LH: Vav3 mediates receptor protein tyrosine kinase signaling, regulates GTPase activity, modulates cell morphology, and induces cell transformation. Mol Cell Biol 2000, 20(24):9212-9224.

34. Sachdev P, Zeng L, Wang LH: Distinct role of phosphatidylinositol 3-kinase and Rho family GTPases in Vav3-induced cell transformation, cell motility, and morphological changes. J Biol Chem 2002, 277(20): I 7638-I7648

35. Houlard M, Arudchandran R, Regnier-Ricard F, Germani A, Gisselbrecht S, Blank U, Rivera J, Varin-Blank $\mathrm{N}$ : Vav I is a component of transcriptionally active complexes. J Exp Med 2002, | 95(9): I | |5-| | 27

36. Movilla N, Bustelo XR: Biological and regulatory properties of Vav-3, a new member of the Vav family of oncoproteins. Mol Cell Biol 1999, I 9(I I):7870-7885

37. Voegel JJ, Heine MJ, Tini M, Vivat V, Chambon P, Gronemeyer H: The coactivator TIF2 contains three nuclear receptor-binding motifs and mediates transactivation through CBP bindingdependent and -independent pathways. Embo J 1998 , I 7(2):507-5 I9.

38. Leo C, Chen JD: The SRC family of nuclear receptor coactivators. Gene 2000, 245(I): I-II

39. Tamas P, Solti Z, Bauer P, Illes A, Sipeki S, Bauer A, Farago A, Downward J, Buday L: Mechanism of epidermal growth factor regulation of Vav2, a guanine nucleotide exchange factor for Rac. J Biol Chem 2003, 278(7):5 |63-5 I 71 .

40. Fan P, Wang J, Santen RJ, Yue W: Long-term treatment with tamoxifen facilitates translocation of estrogen receptor alpha out of the nucleus and enhances its interaction with 
EGFR in MCF-7 breast cancer cells. Cancer research 2007, 67(3): $1352-1360$.

41. Marquez DC, Chen HW, Curran EM, Welshons WV, Pietras RJ: Estrogen receptors in membrane lipid rafts and signal transduction in breast cancer. Molecular and cellular endocrinology 2006, 246(I-2):9I-100.

\section{Pre-publication history}

The pre-publication history for this paper can be accessed here:

http://www.biomedcentral.com/1471-2407/8/158/pre pub

Publish with Bio Med Central and every scientist can read your work free of charge

"BioMed Central will be the most significant development for disseminating the results of biomedical research in our lifetime. " Sir Paul Nurse, Cancer Research UK

Your research papers will be:

- available free of charge to the entire biomedical community

- peer reviewed and published immediately upon acceptance

- cited in PubMed and archived on PubMed Central

- yours - you keep the copyright

Submit your manuscript here:

http://www.biomedcentral.com/info/publishing_adv.asp 